\title{
Feasibility Identification and Computational Efficiency Improvement for Two-Stage RUC with Multiple Wind Farms
}

Zhang, Menglin; Ai, Xiaomeng; Fang, Jiakun; Shuai, Hang; Yao, Wei; He, Haibo; Wu, Qiuwei; Wen, Jinyu

Published in:

IEEE Transactions on Sustainable Energy

Link to article, DOI:

10.1109/TSTE.2019.2936581

Publication date:

2020

Document Version

Peer reviewed version

Link back to DTU Orbit

Citation (APA):

Zhang, M., Ai, X., Fang, J., Shuai, H., Yao, W., He, H., Wu, Q., \& Wen, J. (2020). Feasibility Identification and Computational Efficiency Improvement for Two-Stage RUC with Multiple Wind Farms. IEEE Transactions on Sustainable Energy, 11(3), 1669 - 1678. https://doi.org/10.1109/TSTE.2019.2936581

\section{General rights}

Copyright and moral rights for the publications made accessible in the public portal are retained by the authors and/or other copyright owners and it is a condition of accessing publications that users recognise and abide by the legal requirements associated with these rights.

- Users may download and print one copy of any publication from the public portal for the purpose of private study or research.

- You may not further distribute the material or use it for any profit-making activity or commercial gain

- You may freely distribute the URL identifying the publication in the public portal 


\title{
Feasibility Identification and Computational Efficiency Improvement for Two-stage RUC with Multiple Wind Farms
}

\author{
Menglin Zhang, Xiaomeng Ai, Member, IEEE, Jiakun Fang, Senior Member, IEEE, Hang Shuai, Wei \\ Yao, Senior Member, IEEE, Haibo He, Fellow, IEEE, Qiuwei Wu, Senior Member, IEEE, Jinyu Wen, \\ Member, IEEE
}

\begin{abstract}
The increasing penetration level of wind power challenges robust unit commitment with feasibilities and high computational burden. To meet these challenges, we propose twofold advances for two-stage robust unit commitment (TS-RUC), aiming at providing feasible solution and efficient decision tool for TS-RUC with multiple wind farms. First, the feasibility identification method is proposed to ensure the tractability of TSRUC. The feasibility boundaries are determined based on values of two sets of introduced slack variables, the wind power curtailment and load shedding. Second, the disjunctive programming is used to improve the computational efficiency of the max-min problem, which is reformulated with convex hull relaxation (CHR) method to reduce constraints embedding binary uncertainty variables. Simulation results on the modified IEEE118 bus system and Henan power grid demonstrate that the proposed improvement for the TS-RUC can be implemented for power systems with multiple wind farms and significant wind power. The feasibility identification can guarantee a feasible solution and the use of the CHR can improve the computational efficiency.
\end{abstract}

Index Terms-- Wind power uncertainty, robust unit commitment, feasibility identification, column-and-constraints generation, convex hull relaxation method

\section{NOMENCLATURE}

\section{Indices}

$i \quad$ index of thermal power units

$j \quad$ index of wind farms

$t \quad$ index of periods

$b \quad$ index of system buses

$l \quad$ index of transmission lines

$m$ index of segments of linearized fuel cost function

\section{Parameters}

$N_{\mathrm{G}} \quad$ The total number of thermal power units

$N_{\mathrm{J}} \quad$ The total number of wind farms

$N_{\mathrm{T}} \quad$ The total number of periods

$N_{\text {B }} \quad$ The total number of system buses

$N_{\mathrm{M}} \quad$ The total number of segments of linearized fuel function

$G_{b} \quad$ The set of thermal power units located at bus $b$

$W_{b} \quad$ The set of wind farms located at bus $b$

$C_{i}^{\mathrm{SU}} \quad$ The start-up cost of thermal power unit $i$

$f_{i}^{\text {min }}$ The fuel cost corresponding to the minimum output level of unit $i$
$K_{i t m} \quad$ The slope of segment $m$ of linearized fuel cost function of unit $i$

$P_{i m}^{\max } \quad$ The maximum value of segment $m$ of linearized fuel function of unit $i$

$C^{\text {wind }}$ The penalty price of wind power curtailment

$C^{\text {load }}$ The penalty price of involuntary load shedding

$g_{l b} \quad$ The distribution factor of the net load at bus $b$ on line $l$

$W_{j t} \quad$ The forecast value of wind farm $j$ at period $t$

$\bar{W}_{j t} \quad$ The difference between the upper bound of the uncertainty set and the prediction value of wind farm $j$ at period $t$

$\underline{W}_{j t} \quad$ The difference between the prediction value and the lower bound of the uncertainty set of wind farm $j$ at period $t$

$d_{b t} \quad$ The forecast value of load at bus $b$ at period $t$

$P_{i}^{\max } / P_{i}^{\min } \quad$ The maximum/minimum output of unit $i$

$U_{i}^{\mathrm{R}} / D_{i}^{\mathrm{R}} \quad$ The upward/downward ramping rate of unit $i$

$t_{\mathrm{up}, i}^{\min } / t_{\mathrm{dn}, i}^{\min } \quad$ The minimum up/down time of unit $i$

$C_{i t}^{\mathrm{ur}} / C_{i t}^{\mathrm{dr}} \quad$ The price of upward/downward reserve capacity for unit $i$ at period $t$

Variables

$I_{i t} \quad$ The binary variable for on/off status of thermal power unit $i$ at period $t$

$u_{i t} \quad$ The binary variable for start-up of unit $i$ at period $t$

$v_{i t} \quad$ The binary variable for shut-down of unit $i$ at period $t$

$P_{i t} \quad$ The scheduled power output of unit $i$ at period $t$

$P_{i t m} \quad$ The power output of segment $m$ for unit $i$ at period $t$

$P_{i t}^{\mathrm{ur}} / P_{i t}^{\mathrm{dr}}$ The scheduled upward/downward reserve capacity of unit $i$ at period $t$

$p_{i t}^{\mathrm{ur}} / p_{i t}^{\mathrm{dr}}$ The deployed upward/downward reserve of unit $i$ at period $t$ under worst scenario of wind power

$L_{b t}^{\text {cur }} \quad$ The involuntary load shedding at bus $b$ at period $t$ under worst scenario of wind power

$W_{j t}^{\text {cur }} \quad$ The wind power curtailment for wind farm $j$ at period $t$ under worst scenario of wind power 
$\tilde{w}_{j t} \quad$ The possible wind power output in actual for wind farm $j$ at period $t$

$Z_{j t}{ }^{+} \quad$ The binary variable to indicate if the wind farm $j$ reaches its upper bound at period $t$

$Z_{j t}{ }^{-} \quad$ The binary variable to indicate if the wind farm $j$ reaches its lower bound at period $t$

\section{INTRODUCTION}

When the penetration level of wind power is low, the system operator can deploy a certain level of reserve in the traditional deterministic unit commitment (DUC). However, the significant growth of wind power has changed this situation [1]-[5]. With more wind power integrated, the system is facing a high level of uncertainty.

To cope with the uncertainties, both stochastic programming [6] and robust optimization [7], as well as their combination [8], [9], has been applied in the day-ahead scheduling. The stochastic unit commitment (SUC) [10] - [12] adopts scenario sets to describe uncertainties. However, its computational burden increases with the number of scenarios [13]-[16]. Compared to the SUC, the robust unit commitment (RUC) usually has the easy-to-obtain set to characterize the uncertainty [17], which avoids the intractable scale by using the robust counterpart instead of a huge number of scenarios. To guarantee full adaptability to the uncertainty, two-stage RUC (TS-RUC) has been proposed, and then widely utilized [18][20]. With the increasing number of wind farms, additional problems emerge for traditional TS-RUC, both in formulation and solution algorithms.

From the formulation perspective, the feasibility cannot always be guaranteed if significant variations are embedded. The existing work assumes that the TS-RUC always has a feasible solution [21], [22]. However, such an assumption does not hold when the system has limited flexibility resources to accommodate all the uncertainties. To identify the infeasible instances, [18] formulates a feasibility sub-problem to generate feasibility cuts that will be added to the master problem in the first stage. However, the feasibility sub-problem cannot guarantee a feasible solution of the first stage at all instances without any slack variables introduced. Further, the slack variables and their penalty terms are introduced to the security constraints in the second-stage optimization to guarantee the nonempty solution space. However, pure mathematical terms cannot give system operators the concrete information without clear physical meaning. For example, even though [23-24] have considered the slack variables, they fail to tell what countermeasures should be taken by the system operators when the slack variables are non-zero.

From the algorithm perspective, when the number of wind farms increases, more binary variables and associated constraints are needed to represent the uncertainties of multiple wind farms, which significantly influences the computational efficiency. Most references utilize the column-and-constraints generation (C\&CG) or Benders algorithms to accelerate [25][27]. Ref. [28] investigates the decentralized algorithm to reduce the computational complexity. The difficulty mainly lies in the nonlinear max-min problem. Refs. [21] and [29] utilize outer approximation $(\mathrm{OA})$ and mountain climbing methods to solve the max-min problem, respectively. However, neither OA nor mountain climbing can guarantee the global optimality of the max-min problem, even though they have high computational efficiency. In contrast, the big-M (BM) [30] method has global optimality when reformulating the max-min problem. However, as the BM reformulates the bilinear terms in each period of each wind farm with four sets of mixed integer constraints, it has the problem of computation scale increasing with the number of wind farms. A fact that has been neglected by most researches is the specific characteristic of bilinear terms in the dual optimization of max-min problem. For a pair of bilinear terms, if their contained dual variables are the same, while their contained binary variables are different, and the sum of the two binary variables is equal to one, then such a pair of bilinear terms can be seen as the disjunctive programming [31] and can be relaxed by the convex hull relaxation (CHR) method. Compared to BM, CHR relaxes a feasible region with tighter solution space and fewer constraints [32, 33]. It provides an alternative solution to encounter the large scale of bilinear terms in TS-RUC with better computational efficiency.

In this paper, we focus on developing an efficient decision tool with feasible solutions for power systems with multiple wind farms. Therefore, the traditional TS-RUC is further developed by improving feasibility identification and computational efficiency. With these two advances, the system operators can guarantee the decision-making tractable and less computation burden. The main contributions of this paper are summarized as follows:

1) penalty terms of two sets of introduced slack variables are deployed into the objective of the second stage of the TSRUC in order to address the feasibility identification of uncertainties. The introduced slack variables with physical meaning enable the solution space nonempty and guarantee the existence of feasible solution in all instances. With the feasibility identification, the system operators can extract a feasibility uncertainty set to quantify the maximally allowed uncertainties in the system, within which the system operators can always have feasible uncertainty balance measures.

2) the computationally efficient optimization technique for the TS-RUC is proposed to reduce the computation burden caused by the increasing number of uncertainty variables and constraints when more wind farms are integrated. The CHR method is utilized to reformulate the bilinear terms matched in pairs. With the proposed solution technique, lots of constraints with binary uncertainty variables are removed. Besides, for the first time, a comprehensive comparison is made among CHR, $\mathrm{BM}$, and OA for TS-RUC with multiple wind farms in perspectives of computational accuracy and efficiency.

The rest of the paper is organized as follows. Section II presents the formulation of the TS-RUC model with feasibility identification. The CHR is combined with the $\mathrm{C} \& \mathrm{CG}$ algorithm to solve the TS-RUC model in Section 3. Numerical results on a modified IEEE 118-bus system and a practical power system are presented in Section IV. Section V concludes.

\section{TWO-STAGE RUC FoRMULATION WITH FEASIBILITY IDENTIFICATION}

In this section, the TS-RUC optimization is formulated. The objective function (1) is to minimize the total operation cost while satisfying all security constraints. The first-stage 
operation cost consists of start-up cost, fuel cost and reserve capacity cost in the base case, and the second-stage cost includes the WPC and LS penalty cost under the worst-case scenario.

$$
\begin{aligned}
& \min \left\{\sum_{t=1}^{N_{\mathrm{T}}} \sum_{i=1}^{N_{\mathrm{G}}}\left(C_{i}^{\mathrm{SU}} u_{i t}+f_{i}^{\mathrm{min}} I_{i t}+\sum_{m=1}^{N_{\mathrm{M}}} P_{i t m} K_{i t m}+C_{i t}^{\mathrm{ur}} P_{i t}^{\mathrm{ur}}+C_{i t}^{\mathrm{dr}} P_{i t}^{\mathrm{dr}}\right)+\right.
\end{aligned}
$$

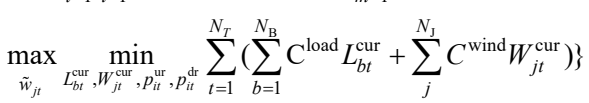

The main difference between the proposed model and those in existing works lies in the objective function at the second stage. The slack variables $W_{j t}^{\text {cur }}$ and $L_{b t}^{\text {cur }}$ are introduced, indicating the WPC and LS, respectively.

Conventionally, the worst-case scenario is assumed to be feasible in the second-stage optimization. However, this may be not true when the penetration level is high. So relaxation for second-stage is a must. Besides, the slack variables in this paper are key performance indices of the power system, which can be used to justify whether the power systems can accommodate all the uncertainties of wind power.

If the determined values of $L_{b t}^{\mathrm{cur}}$ and $W_{j t}^{\mathrm{cur}}$ are zero, then it will be always feasible to accommodate the wind power within the given uncertainty set. Otherwise, with the nonzero values of slack variables, the feasibility boundaries can be derived by subtracting the slack variable values under each critical scenario from the original uncertainty set.

The constraints include (2)-(10) under the base case and (11)-(15) under various uncertainties of wind power.

(2)-(3) model the changes of the on/off status of power plants and impose their minimum up and downtime requirements. (4) constitutes the set of power balance. (5) limits the maximum and minimum power outputs. (6)-(7) describe the linear piecewise constraints of power outputs. The ramping limits, transmission line power flow limits and reserve capacity limits are enforced by (8), (9), (10), respectively. It is worth noting that the active power loss of lines is neglected because we utilize the direct current power flow method to model constraints of the transmission line power flow. (11) describes the power rebalance adapting to wind power uncertainty. (12) guarantees the generation and ramping limits after devoting the reserves in response to wind power uncertainty. (13) represents line flow limits in response to wind power uncertainty. (14) declares the limits of WPC and LS. (15) is the uncertainty set of the wind power by using binary variables. Without loss of brevity, the uncertainty only stems from the wind power production.

$$
\begin{gathered}
\left\{\begin{array}{l}
u_{i t}-v_{i t}=I_{i t}-I_{i t-1} \\
u_{i t}+v_{i t} \leq 1
\end{array}\right. \\
\left\{\begin{array}{l}
I_{i t}-I_{i t-1} \leq I_{i \tau}, \forall \tau \in\left[t+1, \min \left\{N_{T}, t+t_{\mathrm{up}, i}^{\min }-1\right\}\right] \\
I_{i t-1}-I_{i t} \leq 1-I_{i \tau}, \forall \tau \in\left[t+1, \min \left\{N_{T}, t+t_{\mathrm{dn}, i}^{\min }-1\right\}\right]
\end{array}\right. \\
\sum_{i=1}^{N_{\mathrm{G}}} P_{i t}+\sum_{j=1}^{N_{\mathrm{J}}} W_{j t}=\sum_{b=1}^{N_{\mathrm{B}}} d_{b t}, t=1,2, \ldots, N_{T} \\
P_{i}^{\min } I_{i t} \leq P_{i t} \leq P_{i}^{\max } I_{i t}, t=1,2, \ldots, N_{T} \\
P_{i t}=\sum_{m=1}^{N_{\mathrm{M}}} P_{i t m}, \forall i, t
\end{gathered}
$$

$$
\begin{aligned}
& 0 \leq P_{i t m} \leq P_{i m}^{\max }, \forall i, t, m \\
& \left\{\begin{array}{l}
P_{i t}-P_{i t-1} \leq U_{i}^{\mathrm{R}}\left(1-u_{i t}\right)+P_{i}^{\max } u_{i t}, \forall i, t \\
P_{i t-1}-P_{i t} \leq D_{i}^{\mathrm{R}}\left(1-v_{i t}\right)+P_{i}^{\max } v_{i t}, \forall i, t
\end{array}\right. \\
& \left\{\begin{array}{l}
\left|\sum_{b=1}^{N_{\mathrm{B}}} g_{l b} P_{b t}^{\mathrm{inj}}\right| \leq f_{l}^{\mathrm{max}}, \forall l \\
P_{b t}^{\mathrm{inj}}=P_{i t}+w_{j t}-d_{b t}+L_{b t}^{\mathrm{cur}}, i \in G_{b}, j \in W_{b}
\end{array}\right. \\
& \left\{P_{i t}^{\mathrm{ur}} \leq \min \left\{U_{i}^{\mathrm{R}} I_{i t}, P_{i}^{\max } I_{i t}-P_{i t}\right\}, \forall i, t\right. \\
& \left\{P_{i t}^{\mathrm{dr}} \leq \min \left\{D_{i}^{\mathrm{R}} I_{i t}, P_{i t}-P_{i}^{\min } I_{i t}\right\}, \forall i, t\right. \\
& \sum_{i=1}^{N_{\mathrm{G}}}\left(p_{i t}^{\mathrm{ur}}-p_{i t}^{\mathrm{dr}}\right)+\sum_{j=1}^{N_{\mathrm{J}}}\left(\tilde{w}_{j t}-W_{j t}-W_{j t}^{\mathrm{cur}}\right)=\sum_{b=1}^{N_{\mathrm{B}}}-L_{b t}^{\mathrm{cur}} \\
& \left(P_{i}^{\mathrm{min}} I_{i t} \leq P_{i t}+p_{i t}^{\mathrm{ur}}-p_{i t}^{\mathrm{dr}} \leq P_{i}^{\max } I_{i t}\right. \\
& \left(P_{i t}+p_{i t}^{\mathrm{ur}}-p_{i t}^{\mathrm{dr}}\right)-\left(P_{i t-1}+p_{i t-1}^{\mathrm{ur}}-p_{i t-1}^{\mathrm{dr}}\right) \leq U_{i}^{\mathrm{R}}\left(1-u_{i t}\right)+P_{i}^{\max } u_{i t} \\
& \left\{\left(P_{i t-1}+p_{i t-1}^{\mathrm{ur}}-p_{i t-1}^{\mathrm{dr}}\right)-\left(P_{i t}+p_{i t}^{\mathrm{ur}}-p_{i t}^{\mathrm{dr}}\right) \leq D_{i}^{\mathrm{R}}\left(1-v_{i t}\right)+P_{i}^{\mathrm{max}} v_{i t}\right. \\
& 0 \leq p_{i t}^{\text {ur }} \leq P_{i t}^{\text {ur }} \\
& 0 \leq p_{i t}^{\mathrm{dr}} \leq P_{i t}^{\mathrm{dr}} \\
& \mid \sum_{b=1}^{N_{\mathrm{B}}} \mathrm{g}_{l b}\left[\left(P_{i t}+p_{i t}^{\mathrm{ur}}-p_{i t}^{\mathrm{dr}}\right)+\left(\tilde{w}_{j t}-W_{j t}^{\mathrm{cur}}\right)-\left(d_{b t}-L_{b t}^{\mathrm{cur}}\right)\right] \leq f_{l}^{\max } \\
& \left\{\begin{array}{l}
0 \leq L_{b t}^{\mathrm{cur}} \leq d_{b t} \\
0 \leq W_{j t}^{\mathrm{cur}} \leq \tilde{w}_{j t}
\end{array}\right. \\
& \tilde{w}_{j t}=W_{j t}+\bar{W}_{j t} Z_{j t}{ }^{+}-\underline{W}_{j t} Z_{j t}{ }^{-}, Z_{j t}{ }^{+}+Z_{j t}{ }^{-} \leq 1
\end{aligned}
$$

In the TS-RUC model, the decision variables are divided into two groups. The first group consists of binary variables $I_{i t}$, $u_{i t}, v_{i t}$, and continuous variables $P_{i t}, P_{i t m}, P_{i t}^{\text {ur }}, P_{i t}^{\mathrm{dr}}$. The binary variables determine the on/off status, start-up and shutdown of the power plants. The continuous variables determine the output scheduling and upward/downward reserve capacity. In general, these decisions are made before the realization of uncertainties and they are robust against any realization of uncertainties in the second stage.

The second-stage decision variables $p_{i t}^{\mathrm{ur}}, p_{i t}^{\mathrm{dr}}, L_{b t}^{\mathrm{cur}}, W_{j t}^{\mathrm{cur}}$, corresponds to the devoted reserves and the volume of LS and WPC. They represent the re-dispatch actions in response to uncertainties.

The above formulation can be recast as the following compact matrix form, which is suitable for developing solving algorithms.

$$
\left\{\begin{array}{l}
\min _{\boldsymbol{X}, \boldsymbol{Z}, \boldsymbol{Y}}\left\{f(\boldsymbol{X})+\max _{\boldsymbol{Z}} \min _{\boldsymbol{Y}} \boldsymbol{c}^{\mathrm{T}} \boldsymbol{Y}\right\} \\
\text { s.t. } \boldsymbol{g}(\boldsymbol{X}) \leq \boldsymbol{b} \\
\boldsymbol{H} \boldsymbol{X}+\boldsymbol{N} \boldsymbol{Y} \leq \boldsymbol{h}-\left(\boldsymbol{E}_{1} \boldsymbol{Z}^{+}-\boldsymbol{E}_{2} \boldsymbol{Z}^{-}\right)
\end{array}\right.
$$

where $\boldsymbol{X}=\left\{\boldsymbol{u}, \boldsymbol{v}, \boldsymbol{I}, \boldsymbol{P}, \boldsymbol{P}^{\mathrm{ur}}, \boldsymbol{P}^{\mathrm{dr}}\right\}$ is the decision vector of the first stage, and $\boldsymbol{Y}=\left\{\boldsymbol{W}^{\mathrm{cur}}, \boldsymbol{L}^{\mathrm{cur}}, \boldsymbol{p}^{\mathrm{ur}}, \boldsymbol{p}^{\mathrm{dr}}\right\}$ is the deterministic decision vector of the second stage, and $\boldsymbol{Z}=\left\{\boldsymbol{Z}^{+}, \boldsymbol{Z}^{-}\right\}$is the uncertain decision vector of second stage. $\boldsymbol{H}$ and $\boldsymbol{N}$ are the coefficient matrices; $\boldsymbol{b}, \boldsymbol{c}, \boldsymbol{h}$ are the coefficient vectors. The first stage is associated with the day-ahead dispatched 
scheduling with objective $f(\boldsymbol{X})$, while in the intra-day, the infeasibility penalty cost is needed with minimal $\boldsymbol{c}^{\mathrm{T}} \boldsymbol{Y}$ subject to the worst-case scenarios. (16.a) represents constraints (2)-(10) which only contains variables of the pre-dispatch stage, (16.b) represents constraints (11)-(15).

\section{PROBlem REFORMULATION}

Due to the increasing deployment of the wind power, the number of wind farms integrated to the power system raises. This challenges the TS-RUC with the explosive number of uncertainty variables and constraints. In this section, we focus on computational efficiency improvement when solving the formulated optimization in the previous section. The improvement comes from two aspects. First, the C\&CG algorithm efficiently decomposes the original problem into a master problem (MP) and a sub-problem (SP). Second, the relaxation of the SP is conducted from the perspective of disjunctive programming. In the first aspect, the SP aims to derive a critical scenario of wind power uncertainty and provides an upper bound for the original problem. Then, new variables and constraints corresponding to the critical uncertainty scenario are added to the MP to obtain a lower bound. The MP and SP are solved iteratively until the gap between the upper and lower bounds is smaller than a predefined tolerance. In the second aspect, the CHR method is utilized to reformulate the SP problem. Compared with the traditional BM method, the CHR method relaxes the feasible region of the SP with fewer constraints embedded with binary variables, which reduces the calculation burden efficiently.

\section{A. Master UC Problem}

The MP in (17) replaces the objective function of the second stage with an auxiliary variable $\eta$. Then the variables to be solved in the MP include the base-case UC scheduling, ED decisions, auxiliary variable $\eta$ and all the additional variables $\boldsymbol{Y}_{l}(l=1,2, \ldots, k)$, where $l$ is the iteration index and $k$ is current number of iterations. The constraint set of the MP consists of (17.a)-(17.c), which represents the first-stage constraints, the optimal cutting planes, and the feasible cutting planes, respectively. The MP iteratively adds new constraints under the critical uncertain scenario represented by $\boldsymbol{Z}_{l}^{+*}$ and $\boldsymbol{Z}_{l}^{-*}$, and derives new decisions on $\boldsymbol{X}$.

$$
\text { MP : }\left\{\begin{array}{l}
\min _{\boldsymbol{X}, \eta, \boldsymbol{Y}_{l}}\{f(\boldsymbol{X})+\eta\} \\
\text { s.t. } \boldsymbol{g}(\boldsymbol{X}) \leq \boldsymbol{b} \\
\eta \geq \boldsymbol{c}^{\mathrm{T}} \boldsymbol{Y}_{\boldsymbol{l}}, \forall l \leq k \\
\boldsymbol{H} \boldsymbol{X}+\boldsymbol{N} \boldsymbol{Y}_{\boldsymbol{l}} \leq \boldsymbol{h}-\left(\boldsymbol{E}_{1} \boldsymbol{Z}_{l}^{+*}-\boldsymbol{E}_{2} \boldsymbol{Z}_{\boldsymbol{l}}^{-*}\right), \forall l \leq k
\end{array}\right.
$$

The MP aims to relax the original optimization model and provides a lower bound for the original problem. Mathematically, it is a standard MILP problem which can be solved by the commercial solvers.

\section{B. CHR reformulation technique for max-min dual problem}

Fix the first-stage decision $\boldsymbol{X}^{*}=\left\{\boldsymbol{u}^{*}, \boldsymbol{v}^{*}, \boldsymbol{I}^{*}, \boldsymbol{P}^{*}, \boldsymbol{P}^{\mathbf{u r}^{*}}, \boldsymbol{P}^{\mathrm{dr}{ }^{*}}\right\}$ in the $k$ th iteration, the SP can be expressed as follows:

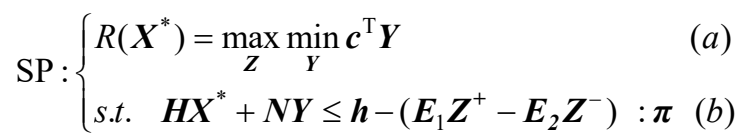

The SP is a typical "max-min" bi-level problem and cannot be solved directly. The duality theory is utilized to transform the inner "min" problem to its equivalent "max" formulation. During the transformation, the variables $\boldsymbol{Z}^{+}$and $\boldsymbol{Z}^{-}$are treated as parameters. Then the max-min problem can be reformulated as a single level "max" problem, as shown in (19).

$$
\left\{\begin{array}{l}
\max _{\boldsymbol{Z}^{+}, \boldsymbol{Z}^{-}, \boldsymbol{\pi}}-\left(\boldsymbol{h}-\boldsymbol{E}_{1} \boldsymbol{Z}^{+}+\boldsymbol{E}_{2} \boldsymbol{Z}^{-}-\boldsymbol{H} \boldsymbol{X}^{*}\right)^{\mathrm{T}} \boldsymbol{\pi} \\
\text { s.t. } \boldsymbol{c}+\boldsymbol{N}^{\mathrm{T}} \boldsymbol{\pi}=0 ; \boldsymbol{\pi} \geq 0 ; \boldsymbol{Z}^{+}, \boldsymbol{Z}^{-} \in\{0,1\}
\end{array}\right.
$$

In (19), the dual problem involves variables $\boldsymbol{Z}^{+}, \boldsymbol{Z}^{-}$, and $\boldsymbol{\pi}$. The objective function contains non-concave bilinear terms $\boldsymbol{Z}^{+} \cdot \boldsymbol{\pi}$ and $\boldsymbol{Z}^{-} \cdot \boldsymbol{\pi}$, which are NP-hard. To linearize these bilinear terms, traditional BM replaces the bilinear terms with auxiliary variables $\boldsymbol{\theta}_{1}$ and $\boldsymbol{\theta}_{2}$ and adds mixed integer constraints on the auxiliary variables, as shown in (20).

$$
\left\{\begin{array}{lr}
\max _{\boldsymbol{Z}^{+}, \boldsymbol{Z}^{-}, \boldsymbol{\pi}}-\left(\boldsymbol{h}-\boldsymbol{H} \boldsymbol{X}^{*}\right)^{\mathrm{T}} \boldsymbol{\pi}+\boldsymbol{E}_{1}^{\mathrm{T}} \boldsymbol{\theta}_{1}-\boldsymbol{E}_{2}^{\mathrm{T}} \boldsymbol{\theta}_{2} \\
\text { s.t. } \quad \boldsymbol{c}+\boldsymbol{N}^{\mathrm{T}} \boldsymbol{\pi}=\mathbf{0}, \boldsymbol{\pi} \geq \mathbf{0}, \boldsymbol{Z}^{+}, \boldsymbol{Z}^{-} \in\{\mathbf{0 , 1}\} \\
0 \leq \boldsymbol{\theta}_{1} \leq \boldsymbol{Z}^{+} M & \text { (a) } \\
0 \leq \boldsymbol{\theta}_{2} \leq \boldsymbol{Z}^{-} M & \text { (b) } \\
\boldsymbol{\pi}-\left(1-\boldsymbol{Z}^{+}\right) M \leq \boldsymbol{\theta}_{1} \leq \boldsymbol{\pi}+\left(1-\boldsymbol{Z}^{+}\right) M & \text { (c) } \\
\boldsymbol{\pi}-\left(1-\boldsymbol{Z}^{-}\right) M \leq \boldsymbol{\theta}_{2} \leq \boldsymbol{\pi}+\left(1-\boldsymbol{Z}^{-}\right) M & \text { (d) }
\end{array}\right.
$$

In (20), $M$ is a large enough constant. The dimensions of $\boldsymbol{Z}^{+}$and $\boldsymbol{Z}^{-}$equal to the product of the number of wind farms $N_{\mathrm{J}}$ and the number of time periods $N_{\mathrm{T}}$. When $N_{\mathrm{J}}$ increases, the dimensions of $\boldsymbol{Z}^{+}$and $\boldsymbol{Z}^{-}$, as well as the number of mixed integer constraints increases. This may significantly affect the calculation time.

The disjunctive programming is an alternative solution for the case that a continuous variable is multiplied by different binary integer variables. Assume that the dual variable $\boldsymbol{\pi}$ is multiplied with binary variables: both $\boldsymbol{Z}^{+}$and $\boldsymbol{Z}^{-}$ simultaneously. The disjunctive programming combines the solution space with multiple discrete constraint sets. The continuous dual variable in (19) can be recast as follows:

$$
\left[\begin{array}{c}
\boldsymbol{Z}^{+} \\
\boldsymbol{g}_{1}(\boldsymbol{\pi}) \geq 0
\end{array}\right] \vee\left[\begin{array}{c}
\boldsymbol{Z}^{-} \\
\boldsymbol{g}_{2}(\boldsymbol{\pi}) \geq 0
\end{array}\right]
$$

where $\boldsymbol{g}_{1}(\boldsymbol{\pi})$ and $\boldsymbol{g}_{2}(\boldsymbol{\pi})$ are constraint sets on $\boldsymbol{\pi}$ corresponding to $\boldsymbol{Z}^{+}$and $\boldsymbol{Z}^{-}$, respectively. As the sum of $\boldsymbol{Z}^{+}$ and $\boldsymbol{Z}^{-}$is equal to 1 , when $\boldsymbol{Z}^{+}=1, \boldsymbol{\pi}$ is uniquely determined by $g_{1}(\pi)$, otherwise, $\pi$ is uniquely determined by $\boldsymbol{g}_{2}(\boldsymbol{\pi})$.

Fig. 1 gives an illustrative example of the disjunctive programming in the two-dimensional solution space. The original feasible region contains two separate parts (marked in red) due to the discrete variables $\boldsymbol{Z}^{+}$and $\boldsymbol{Z}^{-}$. The optimal solution falls into the bottom area when $\boldsymbol{Z}^{+}$equals to 1 , while falls into the top area when $\boldsymbol{Z}^{-}=1$. Our approach is to build a convex hull to cover and connect the two areas in the plan, hence called convex hull relaxation (CHR). By using the CHR method, the feasible region is relaxed to the area within the 
dotted line. It is represented by the smallest set of points that include the full feasible region of the original problem.

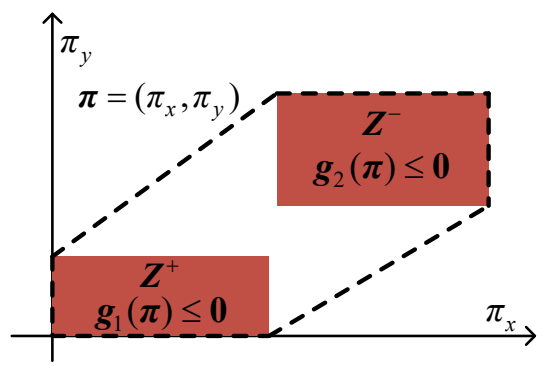

Fig. 1. Relaxation feasible region by CHR method.

In the BM method, each bilinear term is reformulated alone, with four groups of mixed integer constraints, as shown in (20.a)-(20.d). Different from the BM method, the CHR method reformulates the bilinear terms matched in pairs. First, the new variables $\boldsymbol{\pi}_{1}$ and $\boldsymbol{\pi}_{2}$, derived from the original variable $\boldsymbol{\pi}$, are introduced, satisfying $\boldsymbol{\pi}=\boldsymbol{\pi}_{1}+\boldsymbol{\pi}_{2} . \boldsymbol{\pi}_{1}$ and $\boldsymbol{\pi}_{2}$ correspond to the feasible region presented by $\boldsymbol{Z}^{+}$and $\boldsymbol{Z}^{-}$, respectively. Besides, $\boldsymbol{\pi}_{1}$ and $\boldsymbol{\pi}_{2}$ are bounded between zero and $M$ multiplied by $\boldsymbol{Z}^{+}$and $\boldsymbol{Z}^{-}$, respectively. The reformulation of (19) based on the CHR method is shown as follows.

$$
\left\{\begin{array}{l}
\max _{\boldsymbol{Z}^{+}, \boldsymbol{Z}^{-}, \boldsymbol{\pi}, \boldsymbol{\pi}_{1}, \boldsymbol{\pi}_{2}}-\left(\boldsymbol{h}-\boldsymbol{H} \boldsymbol{X}^{*}\right)^{\mathrm{T}} \boldsymbol{\pi}+\boldsymbol{E}_{1}^{\mathrm{T}} \boldsymbol{\pi}_{1}-\boldsymbol{E}_{2}^{\mathrm{T}} \boldsymbol{\pi}_{2} \\
\text { s.t. } \boldsymbol{c}+\boldsymbol{N}^{\mathrm{T}} \boldsymbol{\pi}=\mathbf{0}, \boldsymbol{\pi} \geq \mathbf{0}, \boldsymbol{Z}^{+}, \boldsymbol{Z}^{-} \in\{\mathbf{0 , 1}\} \\
0 \leq \boldsymbol{\pi}_{1} \leq \boldsymbol{Z}^{+} M \\
0 \leq \boldsymbol{\pi}_{2} \leq \boldsymbol{Z}^{-} M \\
\boldsymbol{\pi}=\boldsymbol{\pi}_{1}+\boldsymbol{\pi}_{2}
\end{array}\right.
$$

As can be seen from (22), when $\boldsymbol{Z}^{-}=0, \boldsymbol{\pi}_{2}$ will be constrained to 0 , and $\pi$ will be entirely determined by $\pi_{1}$; when $\boldsymbol{Z}^{+}=0, \boldsymbol{\pi}_{1}$ will be constrained to 0 , and $\boldsymbol{\pi}$ will be entirely determined by $\boldsymbol{\pi}_{2}$.

Mathematically, the reformulated problem in (22) by the CHR method is a MILP problem and can be solved by the commercial solvers. In terms of calculation scale, it adds two groups of mixed integer constraints and a group of linear constraint. Therefore, its calculation scale is less than the BM.

\section{Solving procedure}

The complete procedure of the solving algorithm for the TS-RUC can be described as

Step 1: Set the lower bound $L B=-\infty$ and upper bound $U B=+\infty$ for MP in (17). Let the iteration index $k=0$. Set the tolerance gap $\varepsilon$ as a very small constant.

Step 2: Solve the MP in (17) and derive the optimal solution $\boldsymbol{X}_{\boldsymbol{k}}^{*}=\left\{\boldsymbol{u}_{\boldsymbol{k}}^{*}, \boldsymbol{v}_{\boldsymbol{k}}^{*}, \boldsymbol{I}_{\boldsymbol{k}}^{*}, \boldsymbol{P}_{\boldsymbol{k}}^{*}, \boldsymbol{P}_{\boldsymbol{k}}^{\mathbf{u r}^{*}}, \boldsymbol{P}_{\boldsymbol{k}}^{\mathrm{d \boldsymbol {r } ^ { * }}}\right\}$ and $\eta^{k^{*}}$, as well as the optimal objective value $L^{k}=f\left(\boldsymbol{X}_{\boldsymbol{k}}^{*}\right)+\eta^{k^{*}}$. Then update the lower bound of MP as $L B=L^{k}$.

Step 3: Fix $\boldsymbol{X}_{k}^{*}$, solve the max-min SP based on the CHR reformulation in (22). Derive its optimal solution
$\left(\boldsymbol{Z}_{\boldsymbol{k}}^{+*}, \boldsymbol{Z}_{\boldsymbol{k}}^{-*}, \boldsymbol{\pi}_{k}^{*}, \boldsymbol{\pi}_{1 k}^{*}, \boldsymbol{\pi}_{2 k}^{*}\right)$, as well as the optimal objective value $U^{k}=-\left(\boldsymbol{h}-\boldsymbol{H} \boldsymbol{X}_{\boldsymbol{k}}^{*}\right)^{\mathrm{T}} \boldsymbol{\pi}_{\boldsymbol{k}}^{*}+\boldsymbol{E}_{1}^{\mathrm{T}} \boldsymbol{\pi}_{1 \boldsymbol{k}}^{*}-\boldsymbol{E}_{2}^{\mathrm{T}} \boldsymbol{\pi}_{2 \boldsymbol{k}}^{*}$. Furthermore, update the upper bound as $U B=\min \left\{U B, f\left(\boldsymbol{X}_{\boldsymbol{k}}^{*}\right)+U^{k}\right\}$.

Step 4: If $U B-L B \leq \varepsilon$, terminate the procedure and return $\boldsymbol{X}_{\boldsymbol{k}}^{*} \quad$ as the final solution. Otherwise, let $\boldsymbol{Y}_{k+1}=\left\{\boldsymbol{W}_{\boldsymbol{k}+1}^{\text {cur }}, \boldsymbol{L}_{\boldsymbol{k}+1}^{\text {cur }}, \boldsymbol{p}_{\boldsymbol{k}+1}^{\text {ur }}, \boldsymbol{p}_{\boldsymbol{k}+1}^{\mathrm{dr}}\right\}$ and add optimal and feasible cutting planes in (17.b) and (17.c) to the MP.

Step 5: Update $k=k+1$ and go back to Step 2.

\section{NUMERICAL CASE STUdiES}

In this section, we use the modified IEEE-118 bus system and a practical power system in China to validate the effectiveness of the proposed TS-RUC formulation and the associated solution algorithm.

The simulations on two test systems are both conducted in 2 aspects. First, the effectiveness of the TS-RUC model is tested in terms of feasibility identification and robustness. Second, the computational efficiency of the solving algorithm is tested as well as the global optimality.

The simulations were conducted on the tower server with Intel Xeon E5-2630@2.20 GHz and 128-GB RAM. The TSRUC optimization was implemented in MATLAB and solved by GUROBI 8.0. The gaps for the MP and SP are all set as 1e4.

\section{A. Test on the modified IEEE 118-bus System}

The modified IEEE 118-bus test system includes 54 thermal units with total capacity of $7220 \mathrm{MW}$ and 186 transmission lines, the detailed data of units and network parameters can be found in [34]. $5300 \mathrm{MW}$ wind farms are connected to bus $10,25,26,37$, and 38 , respectively. 13 transmission lines are added to the original system. The upward and downward reserve capacity price of each thermal unit is set as $40 \%$ of its maximum incremental cost of producing energy [35]. The 24-hour wind power generation is based on the historical data of the EirGrid [36], and the load demand profile is based on a provincial power grid of China. Both the wind power and load data have been scaled down to match the system.

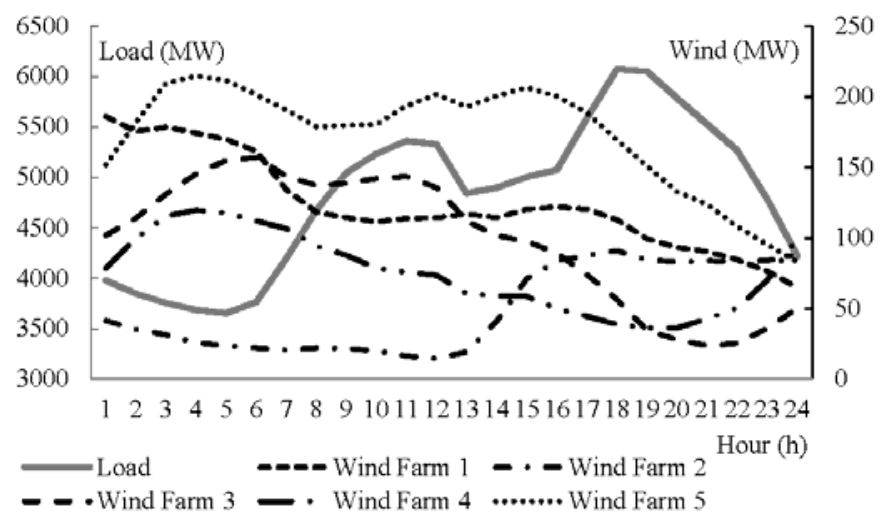

Fig. 2. System load profile and wind power generation of five wind farms.

\section{(1) Feasibility identification and robustness test}

3 studied cases are listed in Table 1. In these cases, the 
capacities of wind power are the same. The difference mainly lies in the ramping rate of the thermal units.

TABLE I

CASE SETTING

\begin{tabular}{cc}
\hline Cases & Simulation parameter \\
\hline Case 1 & Base case \\
\hline Case 2 & $2 \%$ ramping rate improved on the basis of Case 1 \\
\hline Case 3 & $5 \%$ ramping rate improved on the basis of Case 1 \\
\hline
\end{tabular}

The TS-RUC model without feasibility identification is also built and solved for comparison. Table 2 presents the comparative results between two models for 3 cases. It can be seen from Table 2 that with the proposed TS-RUC model we can always identify whether the system can accommodate all the wind power according to the value of WPC and LS. As in Case 3, both the WPC and LS are zero, which indicates that the system has sufficient flexibility to cope with any possible fluctuating scenarios of the wind power. While in Case 1 and Case 2, WPC and LS are not zero, indicating that the system needs more flexibility sources to avoid curtailment or unsupplied load. In contrast, the TS-RUC model without feasibility identification may be intractable in Case 1 and Case 2 , even though it can give the same results as the proposed TSRUC model in Case 3.

TABLE II

COMPARISON RESULTS BETWEEN TWO MODELS

\begin{tabular}{ccccc}
\hline Model & Cases & $\begin{array}{c}\text { Obj. of } \\
\text { first-stage } \\
\left(10^{6} \$\right)\end{array}$ & $\begin{array}{c}\text { Total LS } \\
(\mathrm{MW})\end{array}$ & $\begin{array}{c}\text { Total WPC } \\
(\mathrm{MW})\end{array}$ \\
\hline $\begin{array}{c}\text { TS-RUC with } \\
\text { feasibility } \\
\text { guarantee }\end{array}$ & Case 1 & 1.611737 & 1.70 & 246 \\
\cline { 2 - 5 } $\begin{array}{c}\text { Case } 2 \\
\text { TS-RUC without } \\
\text { feasibility } \\
\text { guarantee }\end{array}$ & Case 3 & 1.608902 & 0.51 & 143 \\
\cline { 2 - 5 } & Case 1 & \multicolumn{3}{c}{ Infeasible } \\
\cline { 2 - 5 } Case 3 & 1.608493 & Infeasible & 0 \\
\hline
\end{tabular}

Case 1 is zoomed in to illustrate the feasibility boundaries of the uncertainty set derived from the proposed TS-RUC model. As shown in Fig. 3, the upper and lower feasibility boundaries can be easily derived by subtracting the WPC and LS from the forecasted maximal available wind power. OUB and OLB are the forecasted upper and lower boundaries, respectively. NUB is the newly derived upper boundary to assess the maximal wind power that the system can accommodate.

It should be noted that the solved first-stage objective and the total amount of WPC and LS are influenced by the price coefficients in the objective function. Different penalty prices of LS and WPC for Case 2 and Case 3 are compared in Table 3. In Case 2, when the penalty prices of LS and WPC are large enough, the first-stage objective remains the same; when the penalty price decreases, the volume of LS and WPC increase to achieve more economical results. In Case 3, when the penalty prices of LS and WPC are decreased to an extent, the volume of LS and WPC in the second stage no longer remains 0 . This phenomenon means that even though the system has the ability to accommodate all the uncertainty of wind power, the WPC and LS contribute to more economical decisions under a smaller penalty price. Therefore, when the proposed model is used, the penalty price of LS and WPC should be set large enough to guarantee its function in feasibility identification.
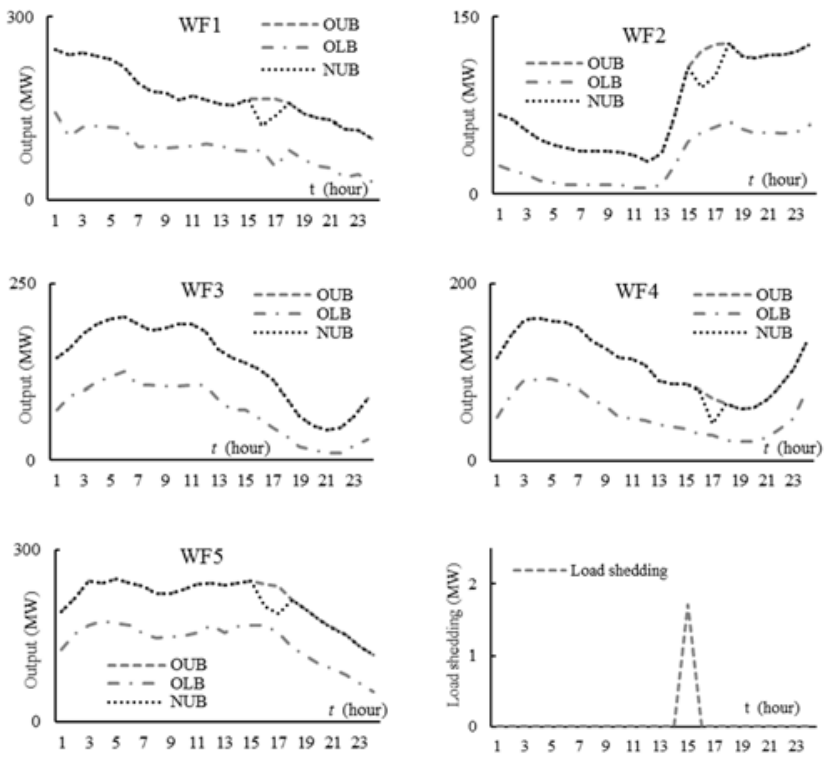

Fig. 3. The derived boundaries of the uncertainty set in Case 1.

TABLE III

COMPARATIVE RESULTS WITH DIFFERENT PENALTY PRICE OF LS AND WPC

\begin{tabular}{ccccc}
\hline Cases & $\begin{array}{c}\text { LS and WPC } \\
\text { price }(\$ / \mathrm{MW} \cdot \mathrm{h})\end{array}$ & $\begin{array}{c}\text { Obj. of first- } \\
\text { stage }\left(10^{6} \$\right)\end{array}$ & $\begin{array}{c}\text { Total LS } \\
(\mathrm{MW})\end{array}$ & $\begin{array}{c}\text { Total WPC } \\
(\mathrm{MW})\end{array}$ \\
\hline \multirow{3}{*}{ Case 2 } & $3.5 \times 10^{7} / 8 \times 10^{5}$ & 1.608903 & 0.51 & 143 \\
\cline { 2 - 5 } & $3.5 \times 10^{6} / 8 \times 10^{4}$ & 1.608903 & 0.52 & 163 \\
\cline { 2 - 5 } & $3500 / 80$ & 1.556829 & 4.56 & 630 \\
\hline \multirow{3}{*}{ Case 3 } & $3.5 \times 10^{7} / 8 \times 10^{5}$ & 1.608493 & 0 & 0 \\
\cline { 2 - 5 } & $3.5 \times 10^{6} / 8 \times 10^{4}$ & 1.608493 & 0 & 0 \\
\cline { 2 - 5 } & $3500 / 80$ & 1.556560 & 2.87 & 449 \\
\hline
\end{tabular}

\section{(2) Robustness of the decision}

In the following, we test the robustness of the solutions in Case 2 and Case 3 by the proposed TS-RUC model. The solutions are obtained with the penalty price of LS and WPC set as $3.5 \times 10^{7}$ and $8 \times 10^{5}$, respectively. By checking whether the volumes of WPC and LS are zero under massive randomly generated scenarios, the robustness can be verified.

In Table 4, we generate two scenario sets to simulate the uncertainty of wind power. Scenario set 1 is generated within the range of the original uncertainty set, while Scenario set 2 is generated within the feasibility boundaries of uncertainty set. Both two sets contain 5000 scenarios. As shown in (18), when the first-stage decisions are fixed and the uncertainty of the wind power is uncovered, the "max-min" problem of the second stage becomes a "min" problem. We solve the "min" problem to achieve WPC and LS under each sampled scenario.

As can be seen from Table 4, when the uncertainty is simulated by Scenario set 1, the maximum and average values of WPC are not zero; while when Scenario set 2 is utilized to simulate the uncertainty, both the WPC and LS are zero in terms of maximum and average values. In Case 3, as its original uncertainty set and feasibility uncertainty set are the same, there are no WPC and LS for both scenario sets. Therefore, it can be concluded that as long as the wind power realization is within the feasibility uncertainty set, the system can always provide a 
feasible re-dispatch solution without WPC and LS.

TABLE IV

ROBUSTNESS TESTING FOR THE SOLUTION OF THE PROPOSED MODEL

\begin{tabular}{|c|c|c|c|}
\hline Scenario sets & Indexes & Case 2 & Case 3 \\
\hline \multirow{4}{*}{ Scenario set 1} & Max WPC (MW) & 30.68 & 0 \\
\hline & Average WPC (MW) & 0.877 & 0 \\
\hline & Max LS (MW) & \multirow{2}{*}{\multicolumn{2}{|c|}{0}} \\
\hline & Average LS (MW) & & \\
\hline \multirow{3}{*}{ Scenario set 2} & Max WPC (MW) & \multirow{2}{*}{\multicolumn{2}{|c|}{0}} \\
\hline & Average WPC (MW) & & \\
\hline & $\frac{\text { Max LS (MW) }}{\text { Average LS (MW) }}$ & \multicolumn{2}{|c|}{0} \\
\hline
\end{tabular}

(3) Computational efficiency

In order to illustrate the advance in the computational efficiency, $\mathrm{BM}$ and $\mathrm{OA}$ methods are implemented and compared. Rather than solving the optimization directly, the inactive constraints are identified and removed first [37, 38]. All the three methods, CHR, BM, and OA are combined with the C\&CG algorithm for the decomposition of the two-stage optimization.

With the increasing number of wind farms integrated into the system, there are more and more stochastic variables in the formulated optimization problem. Hence the size of the maxmin problem in Section 3(b) raises. To investigate the performance of three methods under different calculation scale, three cases are set as shown in Table 5 with a different number of wind farms. In Case 4.1, only one wind farm located at bus 10 is considered. In Case 4.2, there are 3 wind farms located on bus 10, 25 and 26, respectively. In Case 4.3, there are 5 wind farms at bus 10, 25, 26, 37, and 38. The ramping rate of Case 4 is the same as Case 1, and the penalty coefficient of LS and WPC are set as $3.5 \times 10^{7}$ and $8 \times 10^{5}$, respectively. The optimal value of the objective function and the computational time are given in Table 5 and Fig. 4.

TABLE V

Performance of Three Solving Algorithms

\begin{tabular}{cccc}
\hline Cases & $\begin{array}{c}\text { Solving } \\
\text { algorithm }\end{array}$ & $\begin{array}{c}\text { Opt. Obj. } \\
\left(10^{6} \$\right)\end{array}$ & $\begin{array}{c}\text { Iterative } \\
\text { times }\end{array}$ \\
\hline \multirow{3}{*}{ Case 4.1 } & CHR & 1.665505 & 6 \\
\cline { 2 - 4 } & $\mathrm{BM}$ & 1.665505 & 6 \\
\cline { 2 - 4 } & $\mathrm{OA}$ & 1.665501 & 8 \\
\hline \multirow{3}{*}{ Case 4.2 } & $\mathrm{CHR}$ & 1.621866 & 7 \\
\cline { 2 - 4 } & $\mathrm{BM}$ & 1.621866 & 7 \\
\cline { 2 - 4 } Case 4.3 & $\mathrm{OA}$ & 1.619545 & 6 \\
\hline & $\mathrm{CHR}$ & 1.611737 & 9 \\
\cline { 2 - 4 } & $\mathrm{BM}$ & 1.611517 & 9 \\
\hline & $\mathrm{OA}$ & 1.603863 & 7 \\
\hline
\end{tabular}

As can be seen from Table 5, the difference in the optimality between C\&CG-CHR and C\&CG-BM is within the tolerance gap. Therefore, it can be concluded that C\&CG-CHR can guarantee the global optimality, just the same as C\&CGBM. In contrast, the C\&CG-OA method usually obtains a smaller objective value than the other two methods due to the local optimality of the max-min problem. For the OA method, the bilinear term in (19) is linearized around intermediate solution points and linear approximations are added to the OA formulation. Since the bilinear term is non-concave, only a local optimum is guaranteed by the OA algorithm [21]. Failing to obtain the worst-case scenario of the max-min problem means the solution cannot guarantee the robustness of the decision, which can be seen from the nonzero average and maximum values of WPC and LS under 5000 random scenarios derived from the feasible uncertainty set in Table 6 .

TABLE VI

ROBUSTNESS TESTING FOR THREE SOLUTION METHOD

\begin{tabular}{cccccc}
\hline Cases & $\begin{array}{c}\text { Solving } \\
\text { algorithm }\end{array}$ & $\begin{array}{c}\text { Max } \\
\text { WPC } \\
(\mathrm{MW})\end{array}$ & $\begin{array}{c}\text { Average } \\
\text { WPC } \\
(\mathrm{MW})\end{array}$ & $\begin{array}{c}\text { Max LS } \\
(\mathrm{MW})\end{array}$ & $\begin{array}{c}\text { Average } \\
\text { LS } \\
(\mathrm{MW})\end{array}$ \\
\hline \multirow{2}{*}{$\begin{array}{c}\text { Case } \\
4.1\end{array}$} & $\mathrm{CHR}$ & 0 & 0 & 0 & 0 \\
\cline { 2 - 7 } & $\mathrm{BM}$ & 0 & 0 & 0 & 0 \\
\hline \multirow{2}{*}{$\begin{array}{c}\text { Case } \\
4.2\end{array}$} & $\mathrm{OA}$ & 10.19 & 2.58 & 0 & 0 \\
\cline { 2 - 7 } & $\mathrm{CHR}$ & 0 & 0 & 0 & 0 \\
\hline \multirow{2}{*}{ Case } & $\mathrm{BM}$ & 0 & 0 & 0 & 0 \\
\cline { 2 - 6 } 4.3 & $\mathrm{OA}$ & 91.24 & 4.02 & 231.10 & 48.89 \\
\cline { 2 - 6 } & $\mathrm{CHR}$ & 0 & 0 & 0 & 0 \\
\cline { 2 - 7 } & $\mathrm{BM}$ & 0 & 0 & 0 & 0 \\
\hline
\end{tabular}

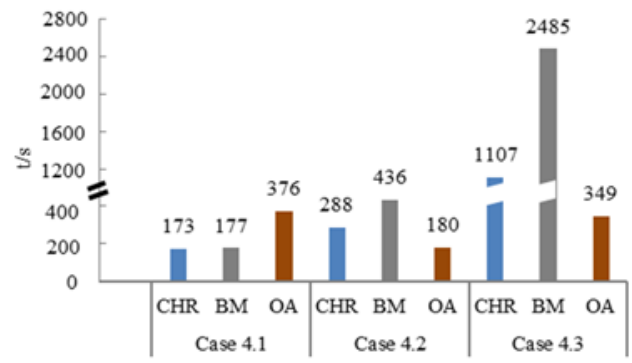

Fig. 4. Calculation time for three solving algorithms.

Fig. 4 shows that the C\&CG-CHR presents a better performance in calculation time than C\&CG-BM, especially in the systems with multiple wind farms. The more wind farms exist in the system, the more improvement can the proposed C\&CG-CHR make. As in Case 4.3, there are 5 wind farms. The C\&CG-BM takes 2,485 seconds to obtain the optimal solution, while C\&CG-CHR can improve the computational efficiency by about $55 \%$ than C\&CG-BM in this case. This is because more wind farms relate to more mixed integer constraints, while the CHR can reformulate the feasible region with fewer constraints embedded with binary variables and connect the discrete constraint set with the minimum convex hull, which can reduce the scale of constraints and shrink the searching region of solution space than BM. For C\&CG-OA method, its iterative times during one solving procedure are random due to the randomly generated uncertainty parameters in each iteration. Therefore its final decision and calculation time are also random, dependent on the randomly generated uncertainty parameters. Even though its calculation time is less than the CHR and BM method in Case 4.3, it is at the expense of losing robustness of solutions.

\section{B. Test on the Practical Henan Power Grid in China}

A practical test system, the Henan power grid in China, is also used to further validate the effectiveness of the proposed TS-RUC formulation and the associated solution algorithm. This system includes 5 wind farms with the total capacity as $3,600 \mathrm{MW}$, and the peak load is 29,622 MW. Besides, this 
system is interconnected with other power systems by 6 tielines with net injected power of 5,821 MW.

Table 7 presents the comparative results between two models for the Henan power grid. Table 7 has similar results with Table 2 . The comparative results validate that the proposed formulation method for the TS-RUC can guarantee the tractability of the model, compared with the traditional TSRUC model.

TABLE VII

COMParison Results BetweEn Two Models For Henan Power Grid

\begin{tabular}{cccc}
\hline Model & $\begin{array}{c}\text { Obj. of first- } \\
\text { stage }\left(10^{7} \$\right)\end{array}$ & $\begin{array}{c}\text { Total WPC } \\
(\mathrm{MW})\end{array}$ & $\begin{array}{c}\text { Total LS } \\
(\mathrm{MW})\end{array}$ \\
\hline $\begin{array}{c}\text { TS-RUC with } \\
\text { feasibility guarantee }\end{array}$ & 1.3737207 & 1099 & 17.7 \\
\hline $\begin{array}{c}\text { TS-RUC without } \\
\text { feasibility guarantee }\end{array}$ & & Infeasible & \\
\hline
\end{tabular}

In addition, the computational performance of the three solution algorithms on the Henan power grid is shown in Table 8. The comparative results demonstrate that the CHR method has higher computational efficiency than the BM method. Even though the OA method has shorter calculation time than the CHR, it cannot guarantee the global optimality, which can be illustrated by comparative results in Table 9 . Table 9 shows the testing results of the robustness of solutions under 5000 random scenarios derived from the feasible uncertainty set. The nonzero values of WPC have verified the non-global optimality of the OA method. In contrast, the CHR method can guarantee the security of the redispatch, with no WPC under tested scenarios. The reason for non-zero values of LS in the CHR is because the randomly generated scenarios derived from the feasible uncertainty set do not consider the load shedding.

TABLE VIII

PERFormance Of THREe SOlving AlgorithmS

\begin{tabular}{ccc}
\hline Solving algorithm & $\begin{array}{l}\text { Obj. of first- } \\
\text { stage. }\left(10^{7} \$\right)\end{array}$ & Calculation time (s) \\
\hline CHR & 1.3737207 & 1449 \\
\hline BM & Fail to give the solution in 8 hours \\
\hline OA & 1.3725859 & 683 \\
\hline
\end{tabular}

TABLE VIIII

RoBustness Testing For THREe SOLVING AlgORITHMS

\begin{tabular}{ccccc}
\hline $\begin{array}{c}\text { Solving } \\
\text { algorithm }\end{array}$ & $\begin{array}{c}\text { Max WPC } \\
(\mathrm{MW})\end{array}$ & $\begin{array}{c}\text { Average } \\
\text { WPC (MW) }\end{array}$ & $\begin{array}{c}\text { Max LS } \\
(\mathrm{MW})\end{array}$ & $\begin{array}{c}\text { Average } \\
\text { LS (MW) }\end{array}$ \\
\hline CHR & 0 & 0 & 12 & 0.42 \\
\hline OA & 736 & 68 & 116 & 0.78 \\
\hline
\end{tabular}

\section{CONCLUSIONS}

With more and more wind farms integrated in the power system, the traditional TS-RUC may suffer from the infeasible solution and high computational burden. This paper proposes two-fold advances for the TS-RUC to address the challenges. For the first advance, the feasibility identification is used to ensure the existence of a feasible solution of the TS-RUC in all instances by determining the feasible boundaries. For the second advance, the CHR method of disjunctive programming is introduced to improve the computational efficiency by reducing the number of constraints embedding binary uncertainty variables. The key findings of the case studies on the modified IEEE 118-bus system and a practical system can be summarized as follows:

- When the uncertainty is in a low level, the TS-RUC with feasibility identification has the same scheduling results with the traditional TS-RUC; when the uncertainty is in a high level, the traditional TS-RUC is infeasible, while the TS-RUC with feasibility identification can guarantee the existence of feasible solution by giving the feasible boundaries of wind power.

- The penalty coefficients of slack variables in the proposed TS-RUC has impact on the model effectiveness. It should be set large enough, usually with higher orders of the objective magnitude, to guarantee the effective identification of the largest boundaries of wind power that can guarantee a feasible solution.

- The computational efficiency is influenced by the number of wind farms both for BM and CHR, due to the increased uncertainty variables and constraints. However, CHR has higher computational efficiency compared to BM, especially when the number of wind farms increases. Besides, CHR is a global optimal method, which can guarantee the reliability of the solution compared to OA.

\section{REFERENCES}

[1] S. Mei, D. Zhang, Y. Wang, F. Liu, and W. Wei, "Robust optimization of static reserve planning with large-scale integration of wind power: A game theoretic approach," IEEE Trans. Sustain. Energy, vol. 5, no. 2, pp. 535-545, Apr. 2014.

[2] M. I. Alizadeh, M. P. Moghaddam, and N. Amjady, "Multistage multiresolution robust unit commitment with non-deterministic flexible ramp considering load and wind variabilities," IEEE Trans. Sustain. Energy, vol. 9, no. 2, pp. 658-670, Apr. 2018.

[3] Y. V. Makarov, P. V. Etingov, J. Ma, Z. Huang, and K. Subbarao, "Incorporating uncertainty of wind power generation forecast into power system operation, dispatch, and unit commitment procedures," IEEE Trans. Sustain. Energy, vol. 2, no. 4, pp. 433-442, Oct. 2011.

[4] H. Shuai, J. Fang, X. Ai, Y. Tang, J. Wen and H. He, "Stochastic optimization of economic dispatch for microgrid based on approximate dynamic programming," IEEE Trans. Smart Grid, 2018, to be published.

[5] H. Nosair, and F. Bouffard, "Flexibility envelopes for power system operational planning," IEEE Trans. Sustain. Energy, vol. 6, no. 3, pp. 800-809, Jul. 2017.

[6] Q. P. Zheng, J. Wang, and A. L. Liu, "Stochastic optimization for unit commitment-A review," IEEE Trans. Power Syst., vol. 30, no. 4, pp. 1913-1924, Jul. 2015.

[7] A. Bental, and A. Nemirovski, "Robust optimization - methodology and applications,". Mathematical Programming, vol. 92, no. 3, pp. 453-480, May 2002.

[8] P. Xiong, P. Jirutitijaroen and C. Singh, "A distributionally robust optimization model for unit commitment considering uncertain wind power generation," IEEE Trans. Power Syst., vol. 32, no. 1, pp. 39-49, Jan. 2017.

[9] C. Duan, L. Jiang, W Fang, and J. Liu, "Data-driven affinely adjustable distributionally robust unit commitment," IEEE Trans. Power Syst., vol. 33, no. 2, pp. 1385-1398, Mar. 2018.

[10] C. Uçkun, A. Botterud, and J. R. Birge, "An improved stochastic unit commitment formulation to accommodate wind uncertainty," IEEE Trans. Power Syst., vol. 31, no. 4, pp. 2507-2517, Jul. 2016.

[11] L. Wu, M. Shahidehpour, and T. Li, "Stochastic security-constrained unit commitment," IEEE Trans. Power Syst., vol. 22, no. 2, pp. 800-811, May 2007.

[12] T. Ding, Q. Yang, X. Liu, C. Huang, Y. Yang, M. Wang, and F. Blaabjerg, "Duality-free decomposition based data-driven stochastic security-constrained unit commitment," IEEE Trans. Sustain. Energy, early access.

[13] Y. Feng, and S. M. Ryan, "Solution sensitivity-based scenario reduction 
for stochastic unit commitment," Computational Management Science, vol. 13, pp. 29-62, 2016.

[14] I. Blanco, and J. M. Morales, "An efficient robust solution to the twostage stochastic unit commitment problem," IEEE Trans. Power Syst., vol. 32 , no. 6, pp. 4477-4488, Nov. 2017.

[15] E. Du, N. Zhang, C. Kang, and Q. Xia, "Scenario map-based stochastic unit commitment," IEEE Trans. Power Syst., vol. 33, no. 5, pp. 46944705, Sep. 2018.

[16] Q. Zhai, X. Li, X. Lei, and X. Guan, "Transmission constrained UC with wind power: an all-scenario-feasible MILP formulation with strong nonanticipativity," IEEE Trans. Power Syst., vol. 32, no. 3, pp. 18051817, May 2017.

[17] Y. Zhang, X. Ai, J. Fang, J. Wen, and H. He, "Data-adaptive robust optimization method for the economic dispatch of active distribution networks," IEEE Trans. Smart Grid, early access, 2018.

[18] R. Jiang, J. Wang and Y. Guan, "Robust unit commitment with wind power and pumped storage hydro," IEEE Trans. Power Syst., vol. 27, no. 2, pp. 800-810, May 2012.

[19] C. Zhao, J. Wang, J. Watson, and Y. Guan, "Multi-stage robust unit commitment considering wind and demand response uncertainties," IEEE Trans. Power Syst., vol. 28, no. 3, pp. 2708-2717, Aug. 2013.

[20] R. Jiang, J. Wang, M. Zhang, and Y. Guan, "Two-stage minimax regret robust unit commitment," IEEE Trans. Power Syst., vol. 28, no. 3, pp. 2271-2282, Aug. 2013.

[21] D. Bertsimas, E. Litvinov, X. A. Sun, J. Zhao, and T. Zheng, "Adaptive robust optimization for the security constrained unit commitment problem," IEEE Trans. Power Syst., vol. 28, no. 1, pp. 52-63, Feb. 2013.

[22] Y. Cho, T. Ishizaki, N. Ramdani, et al., "Box-based temporal decomposition of multi-period economic dispatch for two-stage robust unit commitment ," IEEE Trans. Power Syst., vol. 34, no. 4, pp. 3109 3118,2019

[23] H. Ye, and Z. Li, "Robust security-constrained unit commitment and dispatch with recourse cost requirement," IEEE Trans. Power Syst., vol. 31, no. 5, pp. 3527-3536, Sep. 2016.

[24] Z. Zhang, Y. Chen, X. Liu, et al., "Two-stage robust security-constrained unit commitment model considering time autocorrelation of wind/load prediction error and outage contingency probability of units," IEEE Access, vol. 7, pp. 25398 - 25408, 2019.

[25] B. Zeng and L. Zhao, "Solving two-stage robust optimization problems using a column-and-constraint generation method," Operations Research Letters, vol. 41, no. 5, pp. 457-461, Sep. 2013.

[26] Y. An and B. Zeng, "Exploring the modeling capacity of two-stage robust optimization: variants of robust unit commitment model," IEEE Trans. Power Syst., vol. 30, no. 1, pp. 109-122, Jan. 2015.

[27] B. Hu and L. Wu, "Robust SCUC considering continuous/discrete uncertainties and quick-start units: A two-stage robust optimization with mixed-integer recourse," IEEE Trans. Power Syst., vol. 31, no. 2, pp. 1407-1419, Mar. 2016

[28] S. Bahrami, M. H.Amini. "A decentralized trading algorithm for an electricity market with generation uncertainty," Appl. Energy, vol. 218, pp. 520-532, 2018.

[29] C. Zhao and Y. Guan, "Unified Stochastic and Robust Unit Commitment," IEEE Trans. Power Syst., vol. 28, no. 3, pp. 3353-3361, Aug. 2013.

[30] Y. Zhang, J. Wang, B. Zeng, et al. "Chance-constrained two-stage unit commitment under uncertain load and wind power output using bilinear benders decomposition," IEEE Trans. Power Syst., vol. 32, no. 5, pp. $3637-3647,2017$.

[31] I. E. Grossmann, and S. Lee, "Generalized convex disjunctive programming: nonlinear convex hull relaxation," Computational Optimization \& Applications," vol. 26, no. 1, pp. 83-100, Oct. 2003.

[32] S. Lee, and I. E. Grossmann, "New algorithms for nonlinear generalized disjunctive programming," Computers \& Chemical Engineering, vol. 24, no. 9, pp. 2125-2141, Oct. 2000.

[33] N. W. Sawaya, and I. E. Grossmann, "A cutting plane method for solving linear generalized disjunctive programming problems," Computers \& Chemical Engineering, vol. 29, no. 9, pp. 1891-1913, Aug. 2005.

[34] 2015. [Online]. Available: http: // motor. ece.iit.edu/ Data/ 118bus_ro.xls.

[35] E. Heydarian-Forushani, M. E. H. Golshan, M. P. Moghaddam, M. Shafie-khah, and J. P. S. Catalão, "Robust scheduling of variable wind generation by coordination of bulk energy storages and demand response," Energy Convers. Manage., vol. 106, pp. 941-950, Dec. 2015.

[36] EirGrid. [Online]. Available: http://www.eirgridgroup.com/how-thegrid-works/
[37] J. Zhan, C. Y. Chung and A. Zare, "A fast solution method for stochastic transmission expansion planning," IEEE Trans. Power Syst., vol. 32, no. 6, pp. 4684-4695, Nov. 2017.

[38] M. Zhang, X. Ai, J. Fang, et al. "A systematic approach for the joint dispatch of energy and reserve incorporating demand response," Appl. Energy, vol. 230, pp. 1279-1291, 2018. 